\title{
Similar Outcomes for Two Anemia Treatment Strategies among Elderly Hemodialysis Patients with Diabetes
}

\author{
Thamer $\mathbf{M}^{1}$, Zhang $\mathbf{Y}^{1}$, Kaufman $\mathrm{J}^{2}$, Cotter $\mathrm{D}^{1 *}$ and Hernán $\mathbf{M A}^{3}$ \\ ${ }^{1}$ Medical Technology and Practice Patterns Institute, 5272 River Road, Suite 500, Bethesda, MD 20816, USA \\ ${ }^{2}$ VA NY Harbor Healthcare System, 423 East 23rd St New York, NY 10010, USA \\ ${ }^{3}$ Departments of Epidemiology and Biostatistics, Harvard School of Public Health, Harvard-MIT Division of Health Sciences \\ and Technology, 677 Huntington Avenue Boston, Massachusetts 02115 USA
}

Received: May 31, 2014; Accepted: July 15, 2014; Published: July 31, 2014

*Corresponding author: Dennis Cotter, Medical Technology and Practice Patterns Institute (MTPPI), 5272 River Road, Suite 500, Bethesda, MD 20816, Tel: 301-652-4005; Fax: 301-652-8335; E-mail: dcott@mtppi.org

\begin{abstract}
Background/Aims: To compare mortality and cardiovascular risk in elderly dialysis patients with diabetes under two clinical strategies of anemia correction: maintaining hematocrit (Hct) between 34.5 and $<39.0 \%$ (high Hct strategy), and between 30.0 and $<34.5 \%$ (low Hct strategy) using intravenous alpha epoetin.
\end{abstract}

Methods: Observational data were used to emulate a randomized trial in which diabetic patients who initiated hemodialysis in 20062008 were assigned to each anemia correction strategy. Inverseprobability weighting was used to adjust for measured timedependent confounding.

Results: Comparing high with low hematocrit strategy, the hazard ratio $(95 \%$ confidence interval) was $1.07(0.83,1.38)$ for allcause mortality and $1.00(0.81,1.24)$ for a composite mortality and cardiovascular endpoint.

Conclusions: Among a cohort of elderly hemodialysis patients with diabetes, no differences were found between the low and high hematocrit strategies. A lower target hematocrit - per current Food and Drug Administration (FDA) guidelines - appears to be as safe as higher targets among this population.

Keywords: ESA; Epoetin; Diabetes; Hemodialysis; Mortality; Cardiovascular outcomes; Marginal structural modeling; Inverse probability weighting

\section{Introduction}

Morbidity and mortality rates for persons with diabetes and chronic kidney disease (CKD) in the US are high [1,2]. One potential area for improvement of patient survival is the management of anemia, the most common complication among dialysis patients. Yet, twenty years after the introduction of erythropoiesisstimulating agents (ESA; including epoetin, epoetin alfa, and darbepoetin) to treat anemia, there is still considerable uncertainty in the nephrology community regarding an optimal hematocrit target among dialysis patients with diabetes as a comorbid condition [3-5]. We conducted an observational analysis of elderly dialysis patients with diabetes to compare their mortality and cardiovascular risk under two clinical strategies of anemia correction: maintaining hematocrit (Hct) between 34.5 and $<39.0 \%$ (high Hct strategy), and between 30.0 and $<34.5 \%$ (low Hct strategy) using intravenous alfa epoetin.

\section{Research design and methods}

We used the same source population and methods that we have previously employed to compare these two strategies in patients with diabetes and cardiovascular disease [6]. In this paper, for the first time, we present results for patients with diabetes regardless of cardiovascular disease. Only one other clinical trial, the Trial to Reduce Cardiovascular Events with Aranesp Therapy (TREAT), [7] examined epoetin use among chronic kidney disease patients with diabetes but our study differs and is novel in two important ways: 1) TREAT enrolled predialysis patients while our study includes end-stage renal disease (ESRD) patients; and 2) TREAT evaluated a near normal hematocrit value ( $>39 \%$ ) versus placebo while our study compared the Food and Drug Administration (FDA)-recommended strategy of hematocrit $<33 \%$ with the commonly used strategy of hematocrit 34-39\%.

We emulate a randomized trial by using observational data from the United States Renal Data System (USRDS), which includes the universe of US dialysis patients. We identified incident hemodialysis patients with diabetes who were 65 years and older and received their first ESRD service in 2006-2008. The inclusion criteria were age 65 years or older and evidence of diabetes after 3 months on dialysis. The exclusion criteria were: history of cancer prior to ESRD (studies suggest that patients with cancer might respond differently to epoetin therapy[8,9] , no epoetin therapy in the first 30 days of outpatient dialysis, kidney transplantation or use of peritoneal dialysis modality or darbepoetin (a derivative form of epoetin used primarily in inpatient settings) before baseline, hematocrit $<24 \%$ at baseline, incomplete baseline covariate values, baseline data inconsistent with the strategies of interest, patients who did not reach hematocrit of $30 \%$ at baseline (non responders), or follow-up started in a nondialysis facility (Supplemental Figure 1). A patient was classified as 'diabetic' if diabetes was reported 
to be the primary cause of renal failure and/or diabetes was listed as a co-morbid condition on the Medical Evidence Form 2728 which is completed when a patient enrolls in the Medicare ESRD program. Follow-up started after completing 3 months of hemodialysis therapy (baseline) and ended at the time of first endpoint, death, potential data errors, or loss to follow-up (30day gap in outpatient dialysis or inpatient claims); which ever happened first, or six months after patient entry.

The two endpoints of interest were all-cause mortality and a composite outcome including death or hospitalization for myocardial infarction (MI), stroke or congestive heart failure (CHF), as identified by International Classification of Diseases (ICD)-9 codes on Medicare hospital claims. These outcomes are similar to those used in two recent epoetin randomized trials $[10,11]$. Data on hematocrit and epoetin therapy were obtained from claims for dialysis services submitted by the dialysis facilities for payment by Medicare. Potential confounders were sociodemographics, body mass index, severity of illness and comorbidities (e.g., cerebrovascular disease, chronic obstructive pulmonary disease), amputation, inability to ambulate, receipt of parenteral iron therapy), dialysis facility characteristics, and predialysis use of ESA therapy. All variables included in this study are listed in Table 1.

We emulated the "per protocol" analysis of a randomized clinical trial that compares the high Hct and low Hct strategies if all subjects had adhered to their baseline strategy throughout the entire follow-up. We classified eligible USRDS patients as following one or both of the following strategies: High Hct Strategy: intravenous epoetin alfa to achieve and maintain hematocrit values of $34.5-<39.0 \%$ or Low Hct Strategy: intravenous epoetin alfa to achieve and maintain hematocrit values of $30.0-<34.5 \%$. Under both the strategies, monthly epoetin dose is changed according to the following rules: i. If previous hematocrit is below the target range, epoetin dose is increased by $\geq 10 \%$;

ii. If previous hematocrit is within target range, epoetin dose is decreased by $<10 \%$ times [hematocrit minus lower end of range] or increased by $<10 \%$ times [upper end of range minus hematocrit];

iii. If previous hematocrit is above the target range, epoetin dose is decreased $\geq 25 \%$ or withheld.

The administration of IV iron during the month after the patient undergoes hemodialysis in a facility not participating in the study (e.g., hospital, hospice, nursing home or home health services) and after epoetin dose was withheld, is left to the discretion of the treating physician. We could not directly evaluate the FDA recommended strategy (target Hct $<33 \%$ ) because only a few patients were treated under this strategy during the study years.

For each month of follow-up, if a patient's data were consistent with both strategies (for example, a patient with hematocrit $<30 \%$ whose epoetin dose was increased by $>10 \%$ ), we created an exact copy of the patient's data for each strategy [12]. Patients assigned to a strategy were artificially censored when their treatment data ceased to be consistent with that strategy.

Because higher epoetin doses are more likely to be prescribed to patients with lower hematocrits who also might be at higher mortality and cardiovascular risk, the estimates from our model needed to be adjusted for the effect of time-dependent confounding by indication [13]. In other words, a technique is needed that addresses the issue of confounding by indication; i.e., whether higher epoetin doses correlate with higher Hct levels or more epoetin resistance and lower achieved Hct levels - a conundrum affecting nearly all observational studies using conventional statistical techniques which adjust for confounding
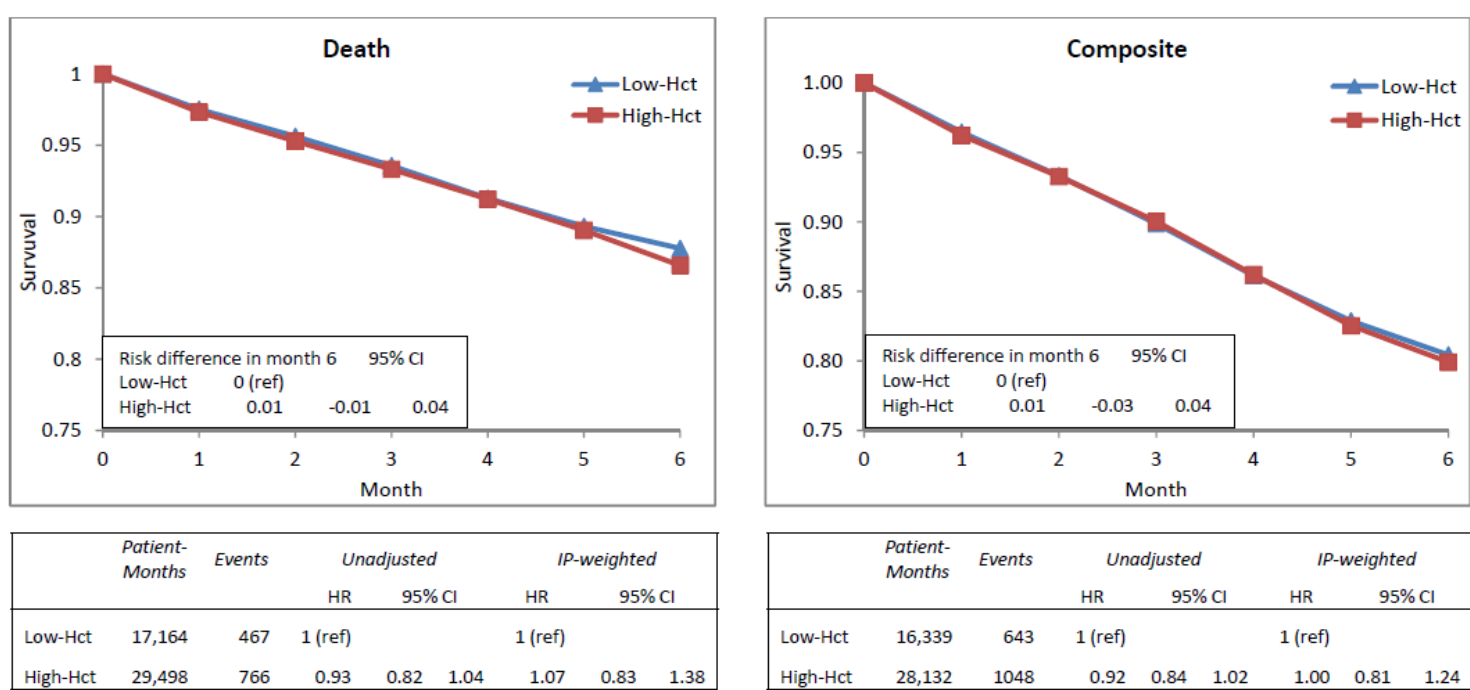

Figure 1: Adjusted survival curves and hazard ratios of death (left column) and composite endpoint (right column) for High $(34.5-<39.0 \%)$ versus Low $(30.0-<34.5 \%)$ Hematocrit Target Treatment Strategies among diabetic patients undergoing hemodialysis using IP-weighted model, United States Renal Data System, 2006-2008. HR=hazard ratio; CI=confidence interval. 
Table 1: Patient characteristics by hematocrit target strategy and diabetic status.

\begin{tabular}{|c|c|c|c|c|}
\hline & \multicolumn{2}{|c|}{ Diabetic } & \multicolumn{2}{|c|}{ Non-diabetic } \\
\hline & Low-Hct & High-Hct & Low-Hct & High-Hct \\
\hline $\mathrm{N}$ & 11,901 & 13,654 & 9,244 & 9,479 \\
\hline Patient Demographics & \multicolumn{4}{|c|}{ Percent } \\
\hline \multicolumn{5}{|l|}{ Age (years) } \\
\hline $65-<70$ & 28.8 & 28.5 & 17.0 & 17.0 \\
\hline $70-<75$ & 27.4 & 26.7 & 20.7 & 20.6 \\
\hline $75-<80$ & 22.7 & 23.1 & 23.0 & 22.8 \\
\hline$\geq 80$ & 21.1 & 21.7 & 39.3 & 39.7 \\
\hline \multicolumn{5}{|l|}{ Sex } \\
\hline Male & 49.6 & 49.2 & 56.0 & 55.6 \\
\hline Female & 50.4 & 50.8 & 44.0 & 44.4 \\
\hline \multicolumn{5}{|l|}{ Race } \\
\hline White & 66.9 & 67.8 & 76.0 & 76.3 \\
\hline Black & 26.9 & 26.3 & 19.6 & 19.8 \\
\hline Other/Unknown & 6.2 & 6.0 & 4.4 & 4.0 \\
\hline \multicolumn{5}{|l|}{ Patient Clinical History } \\
\hline \multicolumn{5}{|l|}{ Total Inpatient days } \\
\hline 0 & 48.0 & 49.3 & 48.5 & 49.6 \\
\hline 1 to 4 & 12.3 & 12.0 & 13.3 & 12.8 \\
\hline 5 to 9 & 19.5 & 19.2 & 18.6 & 18.4 \\
\hline$\geq 10$ & 20.1 & 19.5 & 19.6 & 19.2 \\
\hline \multicolumn{5}{|c|}{ Body Mass Index $\left(\mathrm{kg} / \mathrm{m}^{2}\right)$} \\
\hline$<23.0$ & 17.6 & 17.4 & 34.9 & 35.4 \\
\hline $23.0-<26.6$ & 22.6 & 22.3 & 28.8 & 28.4 \\
\hline $26.6-<31.3$ & 28.1 & 28.0 & 21.7 & 21.6 \\
\hline$>=31.3$ & 31.6 & 32.3 & 14.6 & 14.5 \\
\hline \multicolumn{5}{|c|}{ Cardiovascular comorbidities ${ }^{\mathrm{a}}$} \\
\hline No & 33.5 & 33.3 & 42.4 & 41.9 \\
\hline Yes & 66.5 & 66.7 & 57.6 & 58.1 \\
\hline \multicolumn{5}{|l|}{ Charlson Index ${ }^{\mathrm{b}}$} \\
\hline$<3$ & 18.9 & 18.5 & 53.0 & 52.0 \\
\hline $3-<6$ & 34.9 & 33.8 & 25.3 & 26.1 \\
\hline $6-<8$ & 25.0 & 25.3 & 10.4 & 10.9 \\
\hline$\geq 8$ & 21.2 & 22.3 & 11.3 & 11.0 \\
\hline \multicolumn{5}{|l|}{ Currrent smoker } \\
\hline No & 97.0 & 97.1 & 95.6 & 95.6 \\
\hline Yes & 3.0 & 2.9 & 4.4 & 4.4 \\
\hline \multicolumn{5}{|c|}{ Alcohol/Drug dependence } \\
\hline No & 99.6 & 99.6 & 99.1 & 99.0 \\
\hline Yes & 0.4 & 0.4 & 0.9 & 1.0 \\
\hline \multicolumn{5}{|l|}{ Other severe conditions ${ }^{c}$} \\
\hline No & 90.2 & 89.4 & 95.1 & 95.0 \\
\hline Yes & 9.8 & 10.6 & 4.9 & 5.0 \\
\hline \multicolumn{5}{|c|}{ Chronic obstructive pulmonary disease } \\
\hline No & \begin{tabular}{|l|}
90.4 \\
\end{tabular} & 90.1 & 89.0 & 88.9 \\
\hline Yes & 9.6 & 9.9 & 11.0 & 11.1 \\
\hline \multicolumn{5}{|c|}{ Patient Anemia Management } \\
\hline \multicolumn{5}{|c|}{ Predialysis ESA use } \\
\hline No & 42.7 & 42.3 & 46.5 & 46.0 \\
\hline Yes & 34.3 & 35.2 & 32.3 & 33.0 \\
\hline Unknown & 22.9 & 22.5 & 21.3 & 21.0 \\
\hline
\end{tabular}

\begin{tabular}{|c|c|c|c|c|}
\hline \multicolumn{5}{|l|}{ Predialysis hematocrit } \\
\hline$<30 \%$ & 20.0 & 19.4 & 17.1 & 16.9 \\
\hline $30-<33 \%$ & 24.6 & 24.1 & 22.4 & 21.6 \\
\hline $33-<36 \%$ & 26.5 & 26.2 & 27.0 & 26.4 \\
\hline$\geq 36 \%$ & 28.9 & 30.3 & 33.5 & 35.0 \\
\hline \multicolumn{5}{|c|}{ Average epoetin dose (units/admin) } \\
\hline$<2,500$ & 23.5 & 26.5 & 24.3 & 27.4 \\
\hline $2,500-<4,000$ & 27.0 & 26.7 & 25.4 & 26.8 \\
\hline $4,000-<6,000$ & 24.3 & 23.9 & 24.8 & 23.6 \\
\hline$\geq 6000$ & 25.3 & 22.9 & 25.5 & 22.2 \\
\hline \multicolumn{5}{|l|}{ Average iron dose $(\mathrm{mg} / \mathrm{wk}) \sim$} \\
\hline$<100$ & 24.2 & 27.1 & 26.2 & 29.1 \\
\hline $100-<150$ & 27.7 & 27.3 & 26.9 & 26.9 \\
\hline $150-<200$ & 24.9 & 24.2 & 24.5 & 23.3 \\
\hline$\geq 200$ & 23.2 & 21.4 & 22.4 & 20.6 \\
\hline \multicolumn{5}{|l|}{ Average hematocrit (\%) } \\
\hline $30.1-<33 \%$ & 16.9 & 17.4 & 17.0 & 17.2 \\
\hline $33-<36 \%$ & 27.3 & 28.7 & 28.5 & 29.4 \\
\hline $36-<39 \%$ & 29.8 & 27.7 & 29.8 & 28.0 \\
\hline$\geq 39 \%$ & 26.0 & 26.2 & 24.7 & 25.5 \\
\hline \multicolumn{5}{|c|}{ Dialysis Facility Characteristics } \\
\hline \multicolumn{5}{|l|}{ Region } \\
\hline Northeast (Networks 1-5) & 23.0 & 23.2 & 24.8 & 25.0 \\
\hline $\begin{array}{l}\text { Southeast (Networks 6-8, } \\
13,14 \text { ) }\end{array}$ & 36.3 & 35.8 & 33.4 & 33.8 \\
\hline Midwest (Networks 9-12) & 22.3 & 23.0 & 23.8 & 23.3 \\
\hline West (Networks 15-18) & 18.4 & 17.9 & 18.0 & 18.0 \\
\hline \multicolumn{5}{|l|}{ Ownership } \\
\hline $\begin{array}{l}\text { DaVita (large, for-profit } \\
\text { chain) }\end{array}$ & 28.4 & 28.3 & 28.4 & 28.5 \\
\hline $\begin{array}{l}\text { Fresenius (large, for-profit } \\
\text { chain) }\end{array}$ & 35.1 & 37.0 & 33.9 & 35.4 \\
\hline $\begin{array}{l}\text { Dialysis Clinic, Inc (large, } \\
\text { nonprofit chain) }\end{array}$ & 3.3 & 3.6 & 3.5 & 3.8 \\
\hline $\begin{array}{l}\text { Medium-size Chain (<99 } \\
\text { facilities) }\end{array}$ & 9.4 & 8.5 & 9.9 & 9.5 \\
\hline $\begin{array}{l}\text { Small Chain/Nonchain }(<25 \\
\text { facilities or independent) }\end{array}$ & 23.8 & 22.6 & 24.2 & 22.7 \\
\hline
\end{tabular}

Note: Low hematocrit (Low-Hct) strategy: treatment with epoetin alfa to target hematocrit value $30.0-<34.5 \%$

High hematocrit (High-Hct) strategy: treatment with epoetin alfa to target hematocrit value $34.5-<39.0 \%$

$\sim$ Data was collected in the first three months before baseline.

a Cardiovascular comorbidities include congestive heart failure, atherosclerotic heart disease, cerebrovascular disease, CVA/TIA, peripheral vascular disease, and other cardiac disease.

b Charlson Index is a tool to assess comorbidity and predict survival that has been adapted for use in ESRD patients.

C Includes amputation, inability to ambulate, and inability to transfer.

factors at baseline only. As previously described, [14] we used inverse probability (IP) weighting to adjust for time-dependent confounding by measured time varying covariates including hematocrit, iron treatment, and hospitalization. Specifically, in contrast to more traditional statistical techniques that cannot 
handle time-dependent confounding and sometimes result in spurious findings, IP results tend to more closely approximate the risks or benefits of an intervention both in terms of direction and magnitude of hazard ratios [15-20].

When the outcome of interest was death only, we also adjusted the model for hospitalization due to CHF, MI, and stroke. Each patient received a time-varying weight inversely proportional to the estimated probability of having his/her own observed epoetin dose history. Specifically, we used stabilized IP weights to adjust for selection bias due to artificial censoring discussed above [21]. We truncated the weights to a maximum value of 20 (with a mean of 1.1 and standard error of 2.5) for statistical efficiency. Finally, we estimated the survival curves under each treatment strategy by using the predicted values of weighted outcome models that included an indicator for treatment strategy, baseline covariates, month of follow-up (cubic splines), and product ("interaction") terms between the treatment strategy indicator and the month variables. Point-wise 95\% confidence intervals for all parameter estimates were calculated via percentile-based non-parametric bootstrap based on 300 full samples. Separate analyses were conducted after including patients who did not reach hematocrit of $30 \%$ at baseline (non responders). All analyses were conducted with Statistical Analysis System (SAS) 9.1 (SAS Institute, Cary, $\mathrm{NC}$ ).

\section{Results}

Of 112,449 elderly dialysis patients who initiated epoetin treatment between January 2006 and December 2008, 29,232 patients met the study eligibility criteria. Of 17,291 (59.2\%) who were diabetic, 3,637 were classified in the Low Hct strategy only, 5,390 in the High Hct strategy only, and 8,264 in both. There were no important differences between Low Hct and High Hct patients in patient characteristics or dialysis facilities in which they received care. During the 6-month follow-up, there were 467 deaths (643 composite events) under the Low Hct strategy and 766 deaths $(1,048$ composite events) under the High Hct strategy. We estimated no difference $(0.0 \%)$ in absolute 6-month survival or composite endpoint between the High Hct and Low Hct strategies (Figure 1). The hazard ratios (HRs) (95\% CI) for the High Hct vs. Low Hct strategy were $1.07(0.83,1.38)$ for death and $1.00(0.81,1.24)$ for the composite event (Figure 1). Patient results are provided in a supplemental electronic appendix.

\section{Conclusions}

Using observational data, we emulated a randomized trial of elderly dialysis patients with diabetes assigned to either a high range hematocrit target $(34.5-<39 \%)$ or a low range (30$<34.5 \%$ ) treatment target. We found no differences in the rates of mortality or the cardiovascular composite between these two clinical strategies.

Our study addressed questions left unanswered by TREAT, a recent randomized trial of diabetic CKD predialysis patients [7] This trial found no cardiovascular or renal benefits and an increased stroke risk for those assigned to a target hematocrit level of $39 \%$ compared with those assigned to rescue treatment for hematocrit levels $<27 \%$. However, TREAT enrolled predialysis diabetic patients and therefore the generalizability of their findings to the dialysis populations is uncertain. Our study included ESRD dialysis patients with diabetes. Second, the 4,038 person TREAT trial was not designed to ascertain the optimal hematocrit range and therefore this question remains unanswered. Last, the TREAT investigators themselves suggest that it is possible that other dosing strategies could be developed to mitigate the risks found in their study that targeted a hematocrit of $39 \%$, while conserving the modest benefits of treatment.

In this research, we used the largest observational data available for ESRD research and appropriate analytic methods to handle time-varying treatments and confounders from complex longitudinal administrative data. There are, however, important study limitations. First, the validity of our estimates depends on the assumption that all confounding factors were correctly measured and included in the model [22]. Since epoetin treatment was not randomized, residual confounding could still remain. Second, our analytic method relies on artificial censoring of patients who deviate from their baseline strategy and thus constrained the analysis to a shorter follow up than TREAT. Finally, study results suggesting that a higher hematocrit target does not confer any additional benefits among elderly hemodialysis patients with diabetes is limited to the following population: patients without a history of cancer, previous renal transplant, or severe anemia (Hct<24\%) at dialysis initiation, who were not hospitalized for stroke, congestive heart failure or myocardial infarction in the first 3 months of dialysis, and who were deemed responders to epoetin therapy (achieving a hematocrit of 30\% at baseline) (Supplemental Figure 1).

Previous randomized trials of epoetin therapy - three in predialysis chronic kidney disease patients, $[7,10,11]$ and one in dialysis patients [23] found increased mortality or no benefits for near normal hematocrit targets. As a result of the randomized trial findings, in June 2011, the FDA changed the labeling of ESAs and recommended that physicians "reduce or interrupt the dose" of epoetin if hematocrit exceeds 33\% among dialysis patients. Given the lack of benefits associated with the higher hematocrit target, our study findings support the most recent FDA advisory for dialysis patients with diabetes.

\section{Acknowledgements}

This study was funded in part by an American Diabetes Association (ADA) Grant 1-11-CT-54 and by NIH grants R01 HL080644 (for MA Hernán's work). The data reported herein have been supplied by the US Renal Data System. The interpretation and reporting of these data are the responsibility of the authors and in no way should be seen as the official policy or interpretation of the US government. Furthermore, there are no disclaimers for any of the authors to report. All authors participated in the design, analysis, interpretation, writing, and/ or editing of this study and have seen and approved the final version. Dr. Mae Thamer, as principal investigator and first author had full access to all of the data in the study and had final responsibility for the decision to submit for publication. There are no conflicts of interest for any of the authors. 
Specifically, M.T. wrote the manuscript and researched the data. Y.Z. contributed to all aspects of the manuscript, especially the results and methods section and researched the data. J.K. reviewed/edited the manuscript and contributed to the introduction, interpretation of results and discussion. D.C. reviewed/edited the manuscript, contributed to the discussion, and researched the data. M.H. reviewed/edited the manuscript and contributed to all aspects of the manuscript.

\section{References}

1. Amin AP, Whaley-Connell AT, Li S, Chen SC, McCullough PA, Kosiborod $\mathrm{MN}$, et al. The synergistic relationship between estimated GFR and microalbuminuria in predicting long-term progression to ESRD or death in patients with diabetes: results from the Kidney Early Evaluation Program (KEEP). Am J Kidney Dis 2013; 61(4 Suppl 2):S12-23. doi: 10.1053/j.ajkd.2013.01.005.

2. Astor BC, Matsushita K, Gansevoort RT, van der Velde M, Woodward M, Levey AS, et al. Lower estimated glomerular filtration rate and higher albuminuria are associated with mortality and end-stage renal disease. A collaborative meta-analysis of kidney disease population cohorts. Kidney Int. 2011; 79(12):1331-40. doi: 10.1038/ki.2010.550

3. Khoshdel A, Carney S, Gillies A, Mourad A, Jones B, Nanra R, et al. Potential roles of erythropoietin in the management of anaemia and other complications diabetes. Diabetes Obes Metab. 2008; 10(1):1-9.

4. Vlagopoulos PT, Tighiouart H, Weiner DE, Griffith J, Pettitt D, Salem $\mathrm{DN}$, et al. Anemia as a risk factor for cardiovascular disease and allcause mortality in diabetes: the impact of chronic kidney disease. J Am Soc Nephrol. 2005; 16(11):3403-10.

5. Tong PC, Kong AP, So WY, Ng MH, Yang X, Ng MC, et al. Hematocrit, independent of chronic kidney disease, predicts adverse cardiovascular outcomes in Chinese patients with type 2 diabetes. Diabetes Care. 2006; 29(11):2439-44.

6. Zhang Y, Thamer M, Kaufman J, Cotter D, Hernán MA. Comparative effectiveness of two anemia management strategies for complex elderly dialysis patients. Med Care. 2014; 52 Suppl 3:S132-9. doi: 10.1097/MLR.0b013e3182a53ca8.

7. Pfeffer MA, Burdmann EA, Chen CY, Cooper ME, de Zeeuw D, Eckardt $\mathrm{KU}$, et al. A trial of darbepoetin-alfa in type 2 diabetes and chronic kidney disease. N Engl J Med. 2009; 361(21):2019-32. doi: 10.1056/ NEJMoa0907845.

8. Henke M, Laszig R, Rübe C, Schäfer U, Haase KD, Schilcher B, et al. Erythropoietin to treat head and neck cancer patients with anemia undergoing radiotherapy. Lancet. 2003; 362(9392):1255-60.

9. Leyland-Jones B, BEST investigators and study group. Breast cancer trial with erythropoietin terminated unexpectedly. Lancet Oncol. 2003; 4(8):459-60.

10. Drüeke TB, Locatelli F, Clyne N, Eckardt KU, Macdougall IC, Tsakiris
D, et al. Normalization of hemoglobin level in patients with chronic kidney disease and anemia. N Engl J Med. 2006; 355(20):2071-84.

11. Singh AK, Szczech L, Tang KL, Barnhart H, Sapp S, Wolfson M, et al. Correction of Anemia with Epoetin Alfa in Chronic Kidney Disease. N Engl J Med 2006; 355:2085-98.

12. HIV-CAUSAL Collaboration, Cain LE, Logan R, Robins JM, Sterne JA Sabin C, et al. When to initiate combined antiretroviral therapy to reduce mortality and AIDS-defining illness in HIV-infected persons in developed countries: an observational study. Ann Intern Med. 2011; 154(8):509-15. doi: 10.7326/0003-4819-154-8-201104190-00001.

13. Hernán MA, Hernández-Díaz S, Robins JM. A structural approach to selection bias. Epidemiology 2004; 15(5):615-25.

14. Robins JM, Hernán MA, Brumback B. Marginal structural models and causal inference in epidemiology. Epidemiology 2000; 11(5):550-60.

15. Hammer SM, Squires KE, Hughes MD, Grimes JM, Demeter LM, Currier JS, et al. A controlled trial of two nucleoside analogues plus indinavir in persons with human immunodeficiency virus infection and CD4 cell counts of 200 per cubic millimeter or less. AIDS Clinical Trials Group 320 Study Team. N Engl J Med. 1997; 337(11):725-33.

16. Kinloch-De Loës S, Hirschel BJ, Hoen B, Cooper DA, Tindall B, Carr A, Saurat JH, et al. A controlled trial of zidovudine in primary human immunodeficiency virus infection. N Engl J Med 1995; 333(7):408-13.

17. Gulick RM, Mellors JW, Havlir D, Eron JJ, Gonzalez C, McMahon D, et al. Treatment with indinavir, zidovudine, and lamivudine in adults with human immunodeficiency virus infection and prior antiretroviral therapy. N Engl J Med 1997; 337(11):734-9.

18. Bodnar LM, Davidian M, Siega-Riz AM, Tsiatis AA. Marginal structural models for analyzing causal effects of time-dependent treatments: An application in perinatal epidemiology. Am J Epidemiol 2004; 159(10):926-34.

19. Joffe MM, Ten Have TR, Feldman HI, Kimmel SE. Model selection, confounder control, and marginal structural models: Review and new applications. Am Stat. 2004; 58:272-279.

20. Hernan MA, Brumback BA, Robins JM. Estimating the causal effect of zidovudine on CD4 count with a marginal structural model for repeated measures. Stat Med 2002; 21(12):1689-709.

21. Hernán MA, Lanoy E, Costagliola D, Robins JM. Comparison of dynamic treatment regimes via inverse probability weighting. Basic Clin Pharmacol Toxicol. 2006; 98(3):237-42.

22. Hernán MA, Hernández-Díaz S, Werler MM, Mitchell AA. Causal knowledge as a prerequisite for confounding evaluation: an application to birth defects epidemiology. Am J Epidemiol 2002; 155(2):176-84.

23. Besarab A, Bolton WK, Browne JK, Egrie JC, Nissenson AR, Okamoto $\mathrm{DM}$, et al. The effects of normal as compared with low hematocrit values in patients with cardiac disease who are receiving hemodialysis and epoetin. N Engl J Med 1998; 339(9):584-90. 


\section{Supplemental Electronic Index}

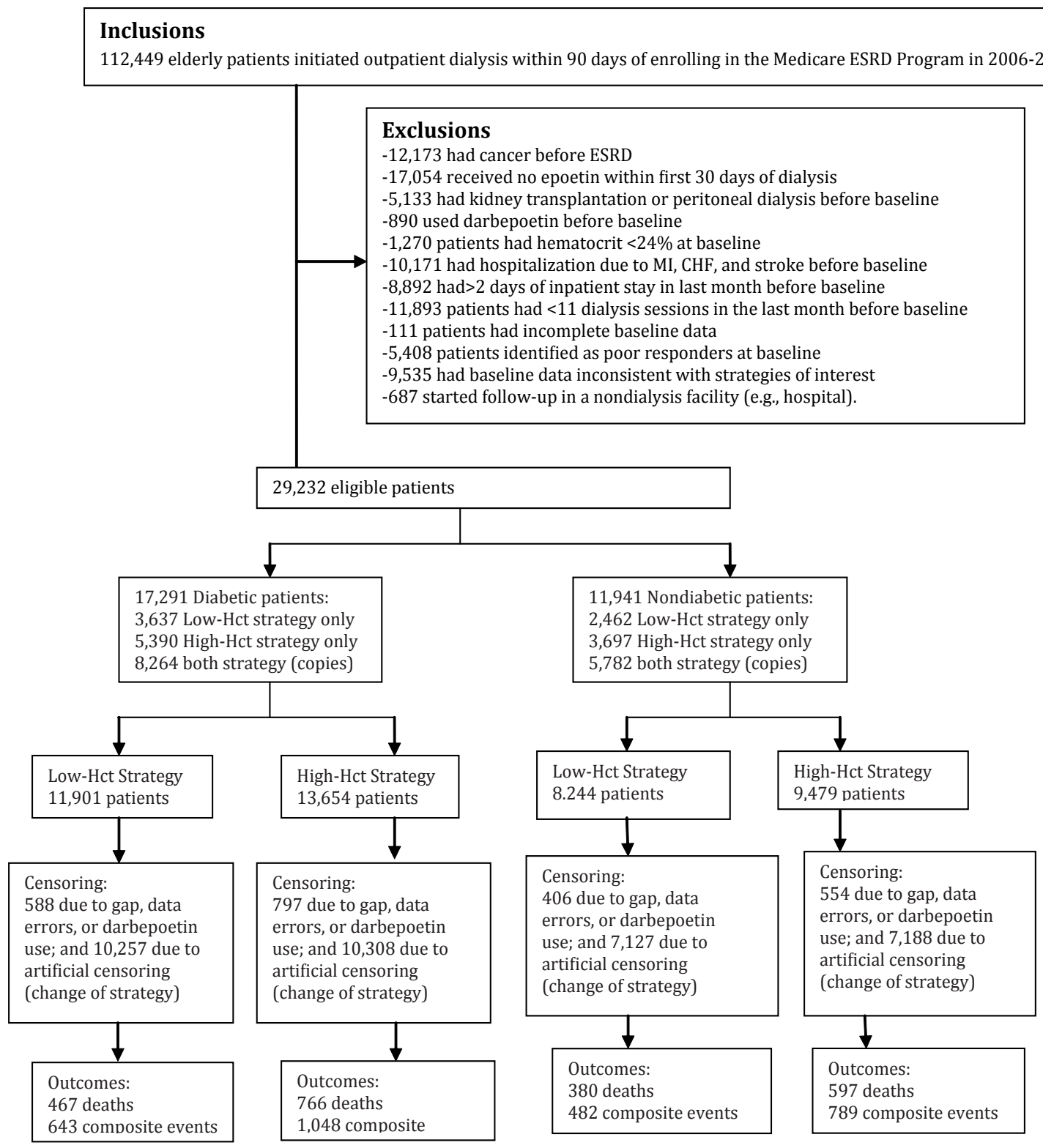

Supplementary file 1: Patient selection and outcomes for an emulated trial of anemia management strategies disaggregated by diabetes status using United States Renal Data System. Low hematocrit (Low-Hct) strategy defined as treatment with epoetin alfa to target hematocrit value $30.0-<34.5 \%$. High hematocrit (High-Hct) strategy defined as treatment with epoetin alfa to target hematocrit value $34.5-<39.0 \%$. 